Original Research Paper

\title{
Colistin Resistance among Gram Negative Organisms; an Evolving Problem from Tertiary Care Hospital, Pakistan 2014
}

\author{
Tuba Bashir and Altaf Ahmed \\ Department of Microbiology, The Indus Hospital, Korangi Crossing, Karachi, Pakistan
}

\author{
Article history \\ Received: 02-06-2015 \\ Revised: 25-02-2016 \\ Accepted: 25-02-2016 \\ Corresponding Author: \\ Tuba Bashir \\ Department of Microbiology, \\ The Indus Hospital, Korangi \\ Crossing, Karachi, Pakistan \\ Off: +92-21-35112709-17; \\ Fax: +92-21-35112718 \\ Email: tuba bashir@yahoo.com; \\ tuba.bashir@tih.org.pk
}

\begin{abstract}
This Study was design to report the evolving Colistin Resistant Gram Negative Organisms from Pakistan in 2014. From 885 isolates of hospitalized patients, 03 Colistin resistant isolates were reported, 02 were Escherichia coli and 01 was Acinetobacter specie. Colistin of all three isolates was tested by Disk diffusion method, MIC by Phoenix 100 (BD) and E-strip. This report contributes a useful addition in literature of Gram Negative Organisms Susceptibility pattern reported from Pakistan, as Colistin is extensively used for the treatment of Multi drug resistant Gram Negative's in hospital settings.
\end{abstract}

Keywords: Colistin Resistance, Gram Negative Organisms, Pakistan

\section{Introduction}

Colistin is bactericidal antibiotic; it binds to lipo polysaccharides and phospholipids of Gram Negative Bacterial Cell membrane. This binding results the intracellular leakage of Cell components and finally cell death (Alfahad and Omrani, 2014). It was introduced in 1952 in clinical practice to treat infections caused by Gram Negative Bacteria (Biswas et al., 2012). In 1970's better antimicrobials was available to treat Gram Negative Infections, as Colistin has poor safety profile (Alfahad and Omrani, 2014).

The Guidelines for Interpreting colistin zone size of Enterobacteriaceae and Acinetobacter specie were not defined by Clinical Laboratory Standard Institute (CLSI), The European Committee on Antimicrobial Susceptibility Testing (EUCAST) and The British Society for Antimicrobial Chemotherapy (BSAC, 2014). CLSI (2014) interpreted the zone diameter for Pseudomonas aeroginosa only (CLSI, EUCAST and BSAC, 2014).

The only reliable Method for Interpreting and reporting of Colistin is MIC method. For Enterobacteriacea and Acinetobacter, the MIC break point of $\leq 2 \mathrm{mg} \mathrm{L}^{-1}$ interpretated as Sensitive by CLSI, EUCAST and BSAC. Peudomonas aeroginosa interpretated as sensitive at $\leq 2 \mathrm{mg} \mathrm{L}^{-1}$ by CLSI and $\leq$ $4 \mathrm{mg} \mathrm{L}^{-1}$ by EUCAST and BSAC (CLSI, EUCAST, BSAC, 2014).

This Study was done in the department of Microbiology, The Indus Hospital, Korangi Crossing,
Karachi, Pakistan. For this Study, the data was analyzed from January 2014 to December 2014.

All Gram Negative Organism which were not intrinsically resistant to Colistin were included in this study. Specimen from wound, pus, fluids, urine, respiratory secretions etc were included:

- Duplicate isolates of the same patient were excluded in this study

- Initially, All Isolates were processed and identified by Standard Conventional method

- For Quality Control Escherichia coli 25922 were used

Colistin Resistant Isolates by Conventional methods were also tested on Phoenix 100 (Becton Dickinson, San Diego, USA). NMIC/ID-94 panels were used for identification and MIC testing of Isolate. Quality Control testing was also performed according to manufacturer's instruction.

Colistin E-test strips (bioMeieux) ranging from 0.06$1024 \mathrm{mg} \mathrm{L}^{-1}$ was also used for further verification of Colistin resistance. Colonies of the tested Isolate were suspended in sterile saline, density of the inoculum were adjusted at 0.5 McFarland standard, Swabbing of inoculum on Muller Hinton Plate (OXOID, UK). E-strip was applied after drying agar surface. Incubate the plates at $37^{\circ} \mathrm{C}$ for $18-24 \mathrm{~h}$.

In 2014, we reported 476 Escherichia coli, 157 Klebsiella specie, 152 Pseudomonas aeroginosa and 100 
Acinetobacter specie in hospitalized patients. Among these 885 Gram Negative Organisms we Isolated 03 Colistin resistant Organisms. 02 were Escherichia coli and 01 was Acinetobacter specie.

The Colistin MIC of all three isolates was reported as $>4 \mathrm{mg} \mathrm{L}^{-1}$ by Automated Phoenix 100. The Tigecycline MIC was $\leq 1 \mathrm{mg} \mathrm{L}^{-1}$ for both Escherichia coli and $2 \mathrm{mg}$ $\mathrm{L}^{-1}$ for Acinetobacter specie by Phoenix 100 .

The E-strip MIC results of Colistin were $8 \mathrm{mg} \mathrm{L}$ for both Escherichia coli and $16 \mathrm{mg} \mathrm{L}^{-1}$ for Acinetobacter specie.

We did not find any in vitro Colistin resistance in Klebsiella specie and Pseudomonas aeroginosa.

The best of our knowledge, this is the 1st report of Colistin resistance among Gram Negative Organisms from Pakistan (Pubmed). Reports of in vitro Colistin resistance has been published from Saudi Arabia and India in recent years (Musa et al., 2013; Abeer et al., 2013) From Greece in vitro colistin resistance was also reported (George et al., 2010). Colistin has been extensively used to treat Multi drug resistant Gram Negative infections (Alfahad and Omrani, 2014).

It has been reported that the growing threat of Colistin resistance might be due to its extensive use as a result of increased Carbapenem resistance among Gram Negative Organisms (Musa et al., 2013).
For clinical laboratories, colistin reporting by disk diffusion method is not recommended, as for Enterobacteriaceae and Acinetobacter spp. no interpretation criteria is available by CLSI. Despite the guidelines criteria MIC testing should also encourage in MDR isolates to improve treatment strategies, leads to better outcomes.

In our study, Table 1 showed that 03 patients were reported as colistin resistant isolates, 02 were Escherichia coli and 01 was Acinetobacter specie. 02 were male patients and 01 was female. All Colistin resistant isolates were sensitive to Tigecycline. 02 were Sensitive only to Tigecycline and Tetracycline whereas, 01 Colistin Resistant Isolate was Sensitive to many other antibiotics including carbapenems. This finding wass also reported in other publications (George et al., 2010). Colistin MIC of all three isolates was very high done by E-strip (Fig. 1).

The limitation of this study was that; we did not perform molecular testing; therefore we are not able to report that which genes are responsible for the Colistin resistance.

On the other hand, there is a great need of MIC reporting of Colistin due to extremely high resistance among Gram Negative's and extensive use of Colistin in hospitalized patients.

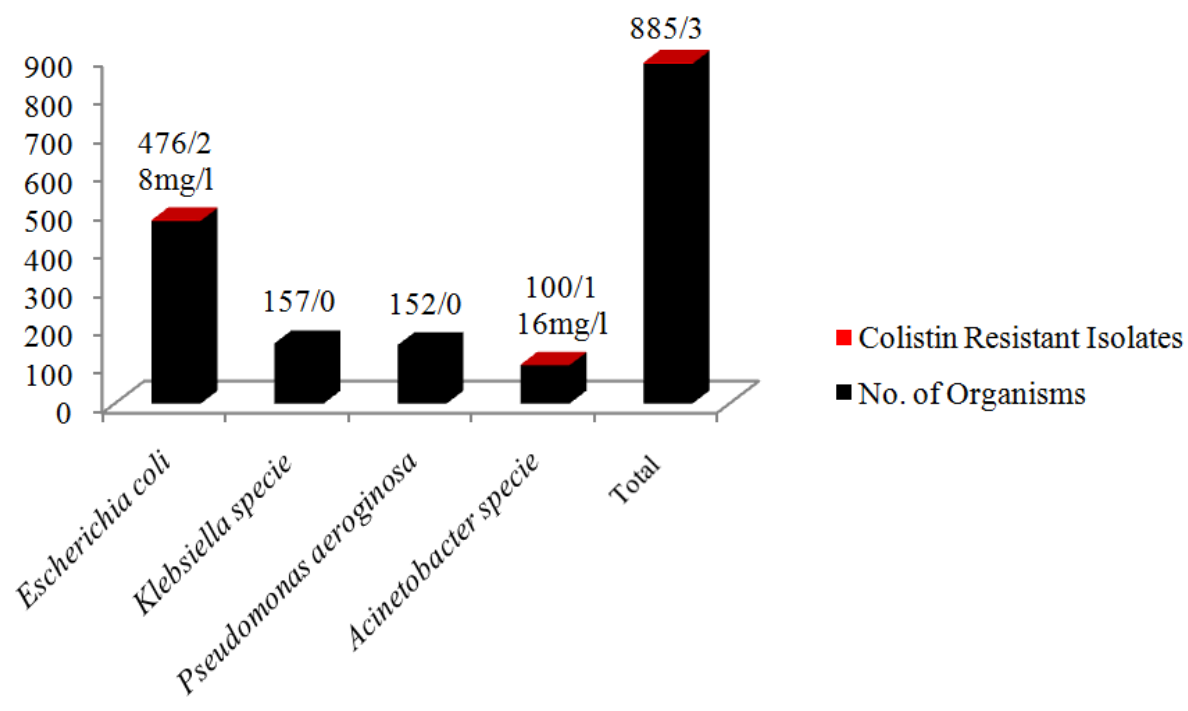

Fig. 1. No. of Colistin Resistant Isolates with MIC by E strip (mg/L) from Total Carbapenem Resistant Isolates in In-patients for the year 2014

Table 1. MIC profile of Colistin resistant isolates by phoenix $100\left(\mathrm{mg} \mathrm{L}^{-1}\right)$

\begin{tabular}{|c|c|c|c|c|c|c|c|c|c|c|c|c|c|}
\hline Isolate/Sex & Age & Sample & AMP & AMC & TZP & CIP & $\mathrm{CN}$ & $\mathrm{AK}$ & IPM & MEM & TGC & SXT & $\mathrm{CT}$ \\
\hline Acine & $44 / \mathrm{F}$ & Urine CS & $>16$ & $>16 / 8$ & $>64 / 4$ & $>2$ & $>8$ & $>32$ & 8 & $>8$ & 2 & $>4 / 76$ & $>4$ \\
\hline Escherichia coli & $25 / \mathrm{M}$ & Urine CS & $>16$ & $>16 / 8$ & $>64 / 4$ & $>2$ & $>8$ & $>32$ & 8 & $>8$ & $\leq 1$ & $>4 / 76$ & $>4$ \\
\hline Escherichia coli & $19 / \mathrm{M}$ & Wound CS & $>16$ & $>16 / 8$ & $\leq 4 / 4$ & $>2$ & $>8$ & $\leq 8$ & $\leq 1$ & $\leq 1$ & $\leq 1$ & $>4 / 76$ & $>4$ \\
\hline
\end{tabular}

Abbreviations for Antibiotics: Amp = Ampicillin; AMC = Amoxicillin-clavulanate; $\mathrm{CIP}=$ Ciprofloxacin; $\mathrm{AK}=\mathrm{Amikacin} ; \mathrm{CN}=$ Gentamicin; SXT = Trimethoprim-Sulfamethoxazole; TZP = Piperacillin/tazobactam; IPM = Imipenem; MEM = Meropenem; TGC $=$ Tigecycline; $\mathrm{CL}=$ Colistin 


\section{Conclusion}

This report is unique, as it is the 1 st in vitro Colistin resistance report from Pakistan. Colistin resistance is an alarming concern because it is used as last resort of treatment in healthcare facilities. Therefore, Strict infection Control guidelines and Antimicrobial Stewardship should implement to overcome the new resistance spread and MIC reporting of MDR isolates should encourage.

\section{Funding Information}

The authors have no support or funding to report.

\section{Author's Contributions}

Both Authors Contributed equally to the Article.

\section{Ethics}

This article is original and contains unpublished material. The corresponding author confirms that all of the other authors have read and approved the manuscript and no ethical issues involved.

\section{References}

Abeer, M.B., I.T. Sahar, A.E.K. Noura and S.O. Ali, 2013. Prevalence of colistin and tigecycline resistance in Acinetobacter baumannii clinical isolates from 2 hospitals in Riyadh Region over a 2year period. Saudi Med. J., 34: 248-53.

PMID: 23475088
Alfahad, W.A. and A.S. Omrani, 2014. Update on colistin in clinical practice. Saudi Med. J., 35: 9-19. PMID: 24445885

Biswas, S., J.M. Brunel, J.C. Dubus, M. ReynaudGaubert and J.M. Rolain, 2012. Colistin: An update on the antibiotic of the 21 st century. Expert Rev. Anti. Infect. Ther., 10: 917-34. PMID: 23030331

BSAC, 2014. British Society for Antimicrobial Chemotherapy Version 13.0, 2014.

CLSI, 2014. Performance Standards for Antimicrobial Susceptibility Testing: M100-S24. 1st Edn., Clinical and Laboratory, ISBN-10: 1562388975, pp: 226.

EUCAST, 2014. European committee on Antimicrobial Susceptibility testing. Miscelleneous Antimicrobials, EUCAST, Clinical MIC.

George, S., K.M. Dimitrios, K. Diamantis, M. Sofia and E.F. Mathew, 2010. In vitro susceptibility to various antibiotics of colistin-resistant gram-negative bacterial isolates in a general tertiary hospital in Crete, Greece. Clin. Infect. Dis., 50: 1689-1691. PMID: 20482269

Musa, AG., B.A. Aref, B. Kamaldeen, S. and Hussam, 2013. Infection due to colistin-resistant Enterobacteriacae in critically-ill patients. J. Infect. Dev. Ctries, 7: 713-719. PMID: 24129623 